\title{
Watt-I-See: Design and Evaluation of an Interactive Installation Using Eco-feedforward Strategies
}

\author{
Filipe Quintal ${ }^{1, *}$, Mary Barreto ${ }^{1}$, Clinton Jorge ${ }^{1}$, VAlentina Nisi $^{1}$ \\ AND NUNO J. NUNES ${ }^{2}$ \\ ${ }^{1}$ Madeira-ITI, Universidade da Madeira, Polo Cientifico e Tecnologico da Madeira, floor -2, \\ Caminho da Penteada, 9020-105 Funchal, Portugal \\ ${ }^{2}$ Madeira-ITI, Técnico - U. Lisboa, Av. Rovisco Pais 1, 1049001 Lisboa, Portugal \\ ${ }^{*}$ Corresponding author: filipe.quintal@m-iti.org
}

\begin{abstract}
In this paper, we present the design and evaluation of Watt-I-See (WISE), a tangible interactive installation aimed at probing awareness in two target audiences (children and adults) about renewable energy resources that could inform eco-feedforward strategies. Our system combines a tangible interface representing five different energy sources, with a set of Lego $^{\mathrm{TM}}$ blocks used to manipulate different weather conditions that affect the available energy mix. Through the evaluation of WISE, our main goal was to expose users to the effort necessary to produce the energy used in our day-to-day lives. We evaluated the installation separately for children and adults. The focus on children was due to their importance in the overall household consumption and their role in future scenarios where consumers will have to adapt to the availability of renewable energy sources. The focus on adults was to observe the impact of such information in their decision making regarding their routines. Our findings highlight the success of the interface in engaging children and adults, creating awareness between weather conditions and energy production and the fossil-fuel energy baseline in the grid. Overall, the study highlights the importance of providing a close point-of-interaction feedback as it enables a better understanding of energy-related decisions, reactions, and availability of resources.
\end{abstract}

\section{RESEARCH HIGHLIGHTS}

- A tangible interactive installation was designed to probe awareness about energy resources.

- The installation was evaluated with two separate types of users: adults and children.

- Users perceived its information as useful and could integrate it into their daily lives.

- New directions for eco-feedback systems that use strategies to control or modify the consumption based on the availability of resources.

Keywords: user studies; tangible interaction; visualization; visualization techniques; novel interaction devices

Handling Editor: Dr. Juan Pablo Hourcade

Received 9 January 2017; Revised 28 August 2017; Editorial Decision 29 August 2017; Accepted 31 August 2017

\section{INTRODUCTION}

Electricity drives our day-to-day lives, it powers our homes, businesses, and services. This indispensability leads to the increase in the global electricity demand (International Energy Outlook 2016, 2016). Consequently, and considering the possible negative impact of the electricity industry in the environment, electricity generation/distribution techniques are being pushed to be cleaner and more efficient. As we stand fuel-based electricity production still accounts for $40 \%$ of the worldwide total (International Energy Outlook 2016, 
2016). However, there are efforts being made to reduce the dependency on fossil-fuels for electricity generation (e.g. European h2020 initiative (European Commission, 2013)). These global initiatives include actions ranging from new legislation to concrete equipment to be deployed into the electricity grid (e.g. batteries).

Considering the human side of electricity sustainability issue, the most frequent approach has been the development and deployment of the so-called Eco-feedback devices (Froehlich et al., 2010; Pierce and Paulos, 2012a). These devices have been defined as technologies used to provide individuals or groups with information about their electricity consumption (Froehlich et al., 2010). These systems can be as simple as a web application or as complex as a whole house custom made monitoring solution. Eco-feedback systems have been proven effective in certain cases with savings in energy consumption between 5\% and 15\% (Fischer, 2008; Pierce and Paulos, 2012a; Quintal et al., 2013b); however, one of the issues in eco-feedback systems is the so-called novelty effect, consumers start to lose interest in the ecofeedback system over time (Nunes et al., 2011; Peschiera et al., 2010; Quintal et al., 2013b), this phenomena could lead to a relapse in the consumption to values prior to the eco-feedback intervention (Peschiera et al., 2010).

\subsection{Eco-feedforward}

Although, eco-feedback devices are nowadays an important tool to save money and control household routines, their role in the household is likely to increase once families' energy needs began to be met by a renewed grid. The new grid technologies combine distributed generation, higher penetration of renewable energy, micro-generation, batteries and vehicleto-grid techniques. In this future scenario, both utilities and consumers will have to adapt to the new situation. In particular, eco-feedback devices will possibly need to include new information and services such as appliance automation, dynamic pricing information, batteries and renewable energy availability and forecast among others.

This new set of services is what we consider ecofeedforward, which we define as the process that controls or modifies energy consumption based on anticipated results or effects of usage patterns and availability of renewable energy with the goal of reducing environmental impact (Kjeldskov et al., 2015; Schrammel et al., 2011; Simm et al., 2015). This concept is directed to a new class of eco-feedback technologies that provide advice or feedback from the anticipated results or effects of individual or group behavior, but also, concerning environmental and availability of resources.

\subsubsection{Contributions}

This work contributes to the eco-feedforward sub-field of eco-feedback. We argue that these services will require the higher involvement of users than the current state of the art eco-feedback systems. Consequently, we designed, implemented and studied Watt-I-See (WISE), an installation inspired by eco-feedforward, where individuals are presented with the real-time resources used to produce their electricity. We take advantage of a closed grid where all the electricity is produced locally and where it is possible to trace in real-time quotas of thermal, wind, hydro and solar electricity in the grid. With this advantage at hand, we envision future scenarios where the energy consumption might have to adapt and plan according to the production at each moment.

This work presents two studies performed to evaluate the WISE system. One was performed with adults, in which, our goal is to move beyond the known limitations of current ecofeedback systems and enhance the understanding of how consumers experience energy production. The second study was performed with a group of children. Through it, we probed children's awareness regarding renewable energy resources and their interaction with the WISE system. The decision of conducting two studies with different demographics was motivated by the body of work which demonstrated that adults and children play an important role in the household dynamics relating energy consumption. Adults are normally responsible for buying the energy consumption appliances and paying the bills. Children were included in this study since research showed the importance of the younger demographic in the household as parents try to educate their children (Barreto et al., 2014; Quintal et al., 2013b). Altogether we do not aim at producing system contributions, since replicating WISE would be very inefficient and difficult. The main focus of this paper is, however, to evaluate an ecofeedforward installation with two different audiences (children and adults). However, our work also contributes in the following topics:

- Assess consumers literacy regarding electricity production.

- Design of a visualization for electricity production.

- Design of eco-feedback systems.

\section{RELATED WORK}

In the following sub-sections, we present three research fields, which motivated us during the development of the presented work. For each area, we present the body of work we find relevant and how our work advances the state of the art.

\subsection{Energy production feedback}

An approach to showcase consumption effects on the environment has been presenting consumers with the source of their electricity. Even though real production data is hard to come by, this concept was tested with micro-generation scenarios 
reporting increased awareness and knowledge of electricity consumption (Filonik et al., 2013; Pierce and Paulos, 2012a). Contributions in this field can be broadly divided into two groups. One where field studies present consumers with information about the availability and forecast for their own energy, such as consumers with micro-generation installation (Kjeldskov et al., 2015; Simm et al., 2015). Another group of exploratory studies, which attempted to perceive how consumers understand electricity, and how to convey the inherent intangibility of electricity for consumers. For this second group, the main goal is to augment electricity information with metadata regarding its quality or origin (Pierce and Paulos, 2013). Ultimately, energy production feedback work has concluded that in general consumers value production information (Kjeldskov et al., 2015; Pierce and Paulos, 2012b). Furthermore, authors claim that this information should be designed in a way that appeals consumers' emotional side, as for instance, consuming their own homegrown and clean energy (Woodruff et al., 2008). The fact is that presenting the raw resources used to produce electricity remains one of the few ways to render electricity, as for example bags of coal or buckets of water (Chetty et al., 2008).

With the present study, we advance the state on energy production feedback of the art with tow novel approaches, by firstly presenting consumers with aggregated production of an island, and secondly by disaggregating the presented production data based on the source of electricity.

\subsection{Eco-feedforward and eco-forecast}

Eco-feedforward and eco-forecast are two similar concepts under the wider eco-feedback umbrella, which enclose techniques to present consumers with information with the goal of changing their future behavior. This information can be the forecast for electricity production (e.g. for consumers with photovoltaic panels) or the prediction of future electricity prices (Fischer et al., 2013). The concept of eco-feedforward was introduced by Yang et al. (2014) in a study with 16 Nest $^{\mathrm{TM}}$ thermostat users; in this study eco-feedforward exemplified as messages that thermostat users could receive to convey the projected impact of following behavior changes recommendations from the system. Kjeldskov et al. (2015) named their eFORECAST contribution as an eco-forecast system which presents expected usage, electricity price, availability of wind power, as well as the expected peaks in demand.

Recent work in these fields is attempting to reduce/remove users' input by implementing automation into the appliances, which are responsible for deciding when to operate based on electricity price or energy availability. One example is the work of Kobus et al. (2015) with 77 households that possessed an energy monitoring system connected to a washing machine, in this system the user had to define ultimate finishing time for the washing cycle, the washing machine was then responsible for finding the best time to start the cycle, in summary, the system made households move their energy consumption to periods with high photovoltaic production, additionally the washing machine was less used in periods with high electricity tariff. A similar study was performed by Bourgeois et al. (2014), where through a set of interventions the authors studied how 18 UK households could better make use of their photovoltaic energy for the same activity. One of the study conditions included a contextual control setting which predicted the best time to start the washing machine cycle, in general users found that this feature could save them time and money, some users even use the information given by this feature to perform other high consumption tasks in the house.

Our contribution in this field is twofold, firstly the evaluation WISE's (the power socket) presents the user with realtime feedback that considers the current renewable quota and the prediction for the next $6 \mathrm{~h}$, which is by definition an ecofeedforward strategy. Secondly, we believe the actual study of consumers' literacy regarding the grid it's valuable for ecofeedforward researchers since it discloses consumers' knowledge of the grid operation.

In the scope of this article, we do not make a differentiation between the eco-feedforward and eco-forecast definitions.

\subsection{Tangible eco-feedback}

Tangible user interfaces have been defined as a special purpose interface for a specific application using explicit physical forms that serve as both input and output (Ishii and Ullmer, 1997). Tangible interaction has shown its efficacy for solving puzzles, programming or even learning abstract concepts such as sounds (Antle et al., 2009; Bakker et al., 2011; Horn et al., 2012). Eco-feedback researchers have adopted tangible interaction strategies to convey resource consumption information to individuals. Pierce and Paulos (Pierce and Paulos, 2013, 2010) discuss several tangible representations for energy, which attempt to provide a tangible representation of electricity in order for it to better relate to consumers. Other researchers have tested more concrete approaches for tangible eco-feedback, for example, the STATIC! Institute has developed several tangible eco-feedback devices. The energy curtain attempts to motivate people to open the curtain and to use more sunlight, by giving feedback as patterns in a window shade woven (Ernevi et al., 2005). Also, from the STATIC! Institute, the PowerCord (Gustafsson and Gyllenswärd, 2005) is as ordinary extension cord redesigned to glow according to the electricity passing by. The Watt-Lite was a tangible display of electricity consumption tested in the workplace (Jönsson et al., 2010) in which, the consumption is displayed by three torches on the floor, representing the realtime consumption and the maximum and minimum for the 
day. In Quintal et al. (2013a) tangible tokens were used to represent individual consumption to the neighbors, a 1-week study disclosed that the feedback had a particular effect in the younger demographic of the household.

Our contribution in this field is the evaluation of a tangible system to present energy production data, how motion can be used to present such data, and finally how adults and children interact with it.

\section{DESIGN OF WATT-I-SEE}

WISE design decisions combine the work from the body of work presented in the last three sub-sections. Firstly, it was driven by studies that presented consumers the energy source, and studies that have displayed the effort needed to produce electricity within the feedback. Secondly, our work was also motivated by studies that took advantage of the inherent qualities of tangible interaction as a means to illustrate complex concepts. Furthermore, the design language followed in WISE was highly influenced and motivated by the work of Pierce and Paulos (2010), in the way we aimed at displaying the intangibility of the electricity by presenting data that is hidden behind our household walls. Regarding the design of the feedback itself our work can be related to Broms and Katzeff work with Watt-Lite and the power cord approach for displaying electricity consumption, in the sense that we employed colors, animations and changes in dimension in the presentation of the electricity information (Broms et al., 2010; Gustafsson and Gyllenswärd, 2005). Our strategy differs, however, from the above-mentioned studies by presenting accurate real-time electricity production from the Madeira Island. In our setup, we explore the information from the local grid consisting of 270 thousand inhabitants in which the grid operates in a closed-circuit system. The island's electricity needs are fulfilled by 2 thermometric power stations, 4 wind parks (located in the mountains), 10 hydroelectric stations (near the base of the mountains), and approximately 700 small and medium-sized photovoltaic installations (dispersed) (more about location at section 4.1). The average yearly electricity production is distributed as follows: $78 \%$ of all energy is produced from thermometric plants, $11 \%$ from hydroelectric stations, $9 \%$ from wind parks and $2 \%$ from dispersed photovoltaic sources. During winter months, the wind and hydro energy source quotas increase significantly, while during dry summer months the renewable quota is significantly lower. The local electricity production quotas are obtained directly from the local energy provider that is responsible for the operation of the electric grid, as well as the producer of most of the electricity consumed locally. Every $15 \mathrm{~min}$ a webservice is updated with the disaggregated quotas for the electricity produced. A script queries the web-service and inserts that data into our database, the production data is then made available through a series of services providing real-time, historical and comparisons production data. Additionally, the local supplier also provides the outcome of a model used to predict the regional production for the next $12 \mathrm{~h}$, this model is used in their internal operation, and we handle this data in a similar manner to the production.

\subsection{Visualization}

WISE resorts to an analogy of 'x-raying' a household wall, exposing the invisible content carried inside pipes and cables that we do not normally get to see, this analogy was highly motivated by artefacts such as the power cord (Gustafsson and Gyllenswärd, 2005) or the power socket (Heller and Borchers, 2011), which attempted to display electricity information by re-imagining the household electricity infrastructure itself (in those cases a power cord and a wall socket). In order to reproduce the pipes metaphor and allow transparency of what is contained inside of them, we resorted to the use of four glass pipes containing a colored vortex, each representing an energy production source available locally: thermometric power stations, wind parks, hydroelectric stations and photovoltaic. The metaphor conveys the idea of different types of energy fueling our household, giving us access to the knowledge of the breakdown and proportions of renewable energies versus fossil. This design decision can be traced to the work of Watt-Lite and nuage vert (Jönsson et al., 2010; 'Reading the clouds,' 2008) and for example where the researchers used size and color to differentiate electricity metrics or to present large aggregate production data.

The glass pipes contain distilled water and liquid colored paraffin. Each vortex is colored to represent a different energy source: dark purple for thermal energy, clear color for the wind, dark blue for hydro and yellow for solar energy source. The size of the vortex ranges from very low (between 1 and $3 \%$ quota) to maximum (these nine levels represent the quota in percentage). The size of the vortices is measured from the top of the tube $0 \mathrm{~cm}$ to the bottom $30 \mathrm{~cm}$. The highest level creates a more aggressive vortex to represent over $91 \%$ (limit selected by average maximum thermal quota) of quota from an individual production source. These levels represent the quota of energy produced and available to final consumers, thus the sum of all four vortices totals $100 \%$ (Fig. 1).

\subsection{The glowing power socket: renewable energy feedback}

In addition to the vortices, the installation is provided with an electricity socket, which we named power socket. The power socket provides additional feedback on the overall quota of renewable energy in the grid. The decision of also implementing feedback in the socket, was motivated by the body of work that recommends that the feedback should be present as close to the point of the decision as possible, additionally the 

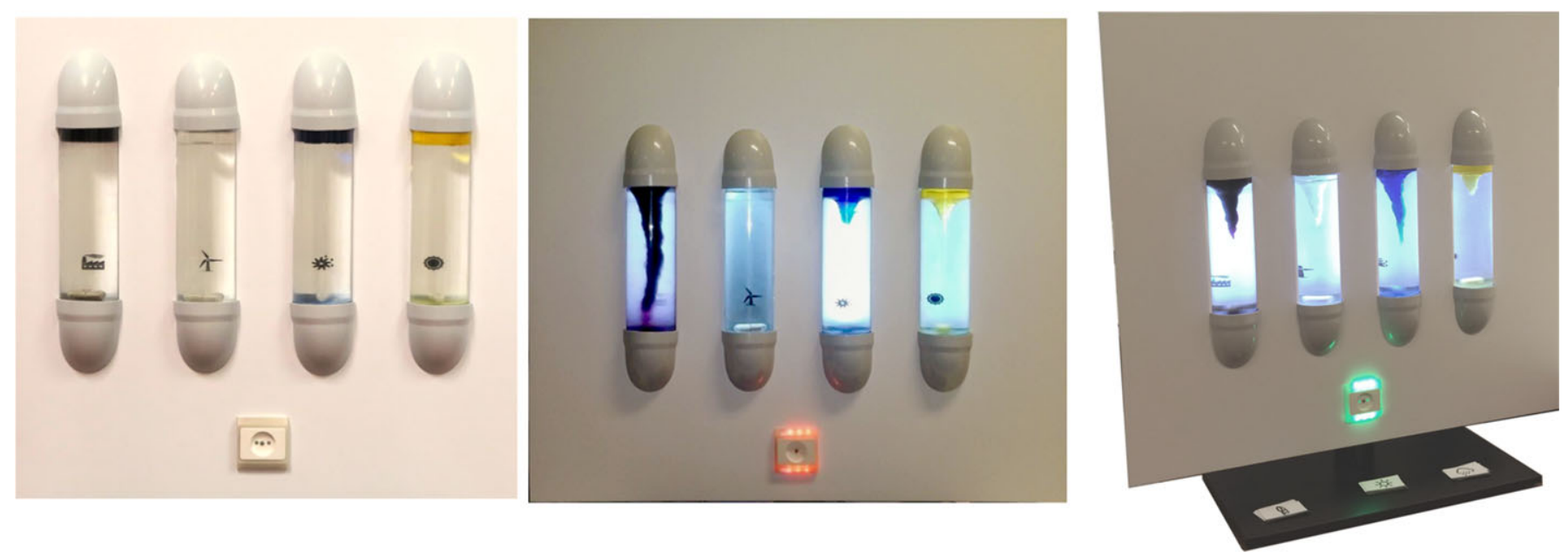

Figure 1. Wise panel, turned off (left), displaying high (middle) and low (right) presence of renewable energy.

power socket is also directly related to Heller work (Heller and Borchers, 2011; Heller et al., 2013), but instead of displaying electricity consumption we propose electricity production information.

The power socket has five possible states (Fig. 2), which vary from green (high percentage of renewable energy in the grid) to red (low percentage of renewable energy in the grid). The selection of which state to present is based on the outcome of a model which uses: (i) current real-time production quotas; (ii) current day averages; (iii) week averages; (iv) month averages; (v) 5-h production prediction quotas. The week and month averages were calculated over historical data from the past 3 years.

It is also important to mention the impact of the electricity production prediction in the power socket presentation. For example, if the current presence of renewable on the grid is low, but there is a forecast for more renewables in the next 5 $\mathrm{h}$ then the system would display a less appealing color on the power socket (orange or red). On the other hand, if the current presence of renewable is low, but the prediction for renewable production is even lower, the system would present a greener power socket.

\subsection{Production visualization}

WISE measures $1.22 \mathrm{~m} \times 0.9 \mathrm{~m}$ (without the stand) built from wood and covered in matte white vinyl. Four glass tubes $(9 \times 30 \mathrm{~cm})$ are used to represent the individual production sources. Vortices are created by DC motors controlled by an Arduino micro-controller. The DC motors rotate a large magnet that subsequently, rotates a magnetic bar inside the glass tube creating the vortex. A smaller magnet and Hall Effect switch is used to calculate the rpm's of each motor in order to leverage the rotation between the different tubes. Additionally, LED strips and drivers are used to retro- illuminate the glass tubes that are covered in tracing paper and contain the printed icons for each energy source (Fig. 1). A computer power supply is used to power the installation.

The electricity socket is a common power socket where the outer bezel was 3D printed using a transparent plastic. The color of the socket is obtained by RGB LEDs.

\subsection{Interaction}

The WISE installation was evaluated with two different groups of people, adults and children, therefore the actual interaction with the installation was different among the groups. However, the logic behind the interaction was the same, individuals (both children and adults) selected weather and time of the day variables which would then affect the presentation in the WISE vortexes and power socket. An application built in Processing communicates with the Arduino microprocessor. This application is responsible for starting/ending the installation as well as changing the presentation displayed.

\subsubsection{Interaction rationale}

The rationale behind the interaction with WISE originated by the fact that in general, consumers cannot control what energy is produced at each moment. At the very best a consumer can predict the weather or other natural phenomena associated with the availability of energy (Chetty et al., 2008; Filonik et al., 2013; Woodruff et al., 2008). This way in the WISE interaction consumers can control several variables related to the time of the day and weather conditions that would affect directly the installation. In both evaluations after a selection was made, WISE would need approximately $10 \mathrm{~s}$ to adjust before settling in the correct visualization. Next, we explain how the interaction with WISE was adapted for children and adults. 


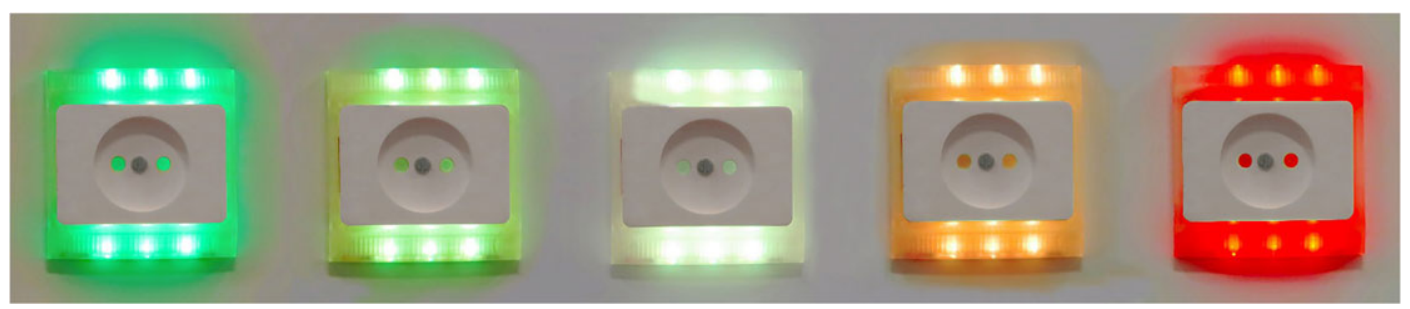

Figure 2. Five possible stages of the Power Socket, from high presence of renewable energy (left) to low (left).

Adults interaction with WISE (EVI). Adults were given a set of cards in which they combine the following variables: rain (4 values) wind (4 values), cloud coverage (4 values) and time of the day (6 values). Participants would then select a combination of the cards and a research member would input those values in a Processing application. This application would then query our database with 2 years' worth weather conditions for the island and select a date where the conditions matched the selected cards (or at least the best fit). Next, the application queries our database with real production data for those 2 years and collected the production quotas for the selected day, those values were finally used to update the WISE panel and power socket.

Children interaction with WISE (EV2). Children interacted with the installation in a simplified manner. Children interacted with the WISE installation by manipulating a set of six small Lego ${ }^{\mathrm{TM}}$ blocks, placed in front of the installation, see Fig. 3 (right). The blocks were used to manipulate weather conditions, stacking them on top of the three icons placed on the table representing, sun, wind, and rain. The sun, rain and wind variables could have three different values (low, medium and high) resulting in nine possible combinations of weather conditions.

The selected weather conditions had a direct relation with displayed energy quotas (rain influenced hydroelectric energy, sun photovoltaic and wind affected wind power). The first Lego $^{\mathrm{TM}}$ block placed on the corresponding weather icon represented $5 \%$ of the total energy production, while the second stacked block represented an additional 15\% (by itself) and the third one an additional 25\% (by itself) of the selected energy, for a maximum total of $45 \%$ per source. The decision for implementing the interaction in this non-linear way was considered after several tests with WISE. Since we were using only 3 blocks the difference between 1 and 2 or 2 and 3 had to be clear, we did not consider the addition of more $\mathrm{Lego}^{\mathrm{TM}}$ blocks as it would have made the interaction cumbersome. Our test showed us that if all the blocks had the same weight then we would need more blocks to represent a more accurate scenario of the local energy production. Either each block would represent too little energy, in that case, there would be no real impact in the visualization or, each block would represent too much energy and it would be easy to overcome the $100 \%$ quota only with renewable energy (which it is not possible at the current stage in the island).

After the participants made a selection, the fossil-fuel energy quota was used to fill up the remaining until $100 \%$ of energy production. For example, one $\mathrm{Lego}^{\mathrm{TM}}$ block placed on the sun icon and two blocks on the rain icon would result in $5 \%$ of photovoltaic energy and $20 \%(15 \%+5 \%)$ of hydroelectric energy produced on the island, while the rest was filled with thermal energy (75\%). If the weather conditions would sum up for more than $100 \%$ we explained to the children that such conditions were impossible to happen all at the same time in real life (for example, lots of wind, rain and sun simultaneously).

\section{EVALUATION METHODOLOGY}

The WISE installation was evaluated in two isolated events to explore both audiences (adults EV1 and children EV2) responses to an eco-feedforward installation. These evaluations aimed to understand: How do adults and children perceive eco-feedforward designed installations? Does a tangible installation help users understand energy forecast and availability? These questions were assessed using questionnaires (designed carefully according to each target involved) and interaction with the installation. About 10 adults and 56 children interacted with it in 2 distinct moments. In this section, we describe how both evaluations proceeded.

\subsection{Participants and procedure EV1}

The first study started with a contact questionnaire of 57 electricity consumers, 35 male, and 22 female, with an average age of 29 (S.D. = 8), from an Island in the south of Europe. Participants were recruited through social networks and wordof-mouth. Ten of the contacted individuals were selected to undertake the remainder of the study and interact physically with WISE at a local research institute. Each participant interacted with WISE individually for the duration of approximately $50 \mathrm{~min}$. The study included free interaction with WISE, a 14 question semi-structured interview recorded through the researcher laptop and a 20 question (five-point Likert scale) survey at the end, this survey addressed how 

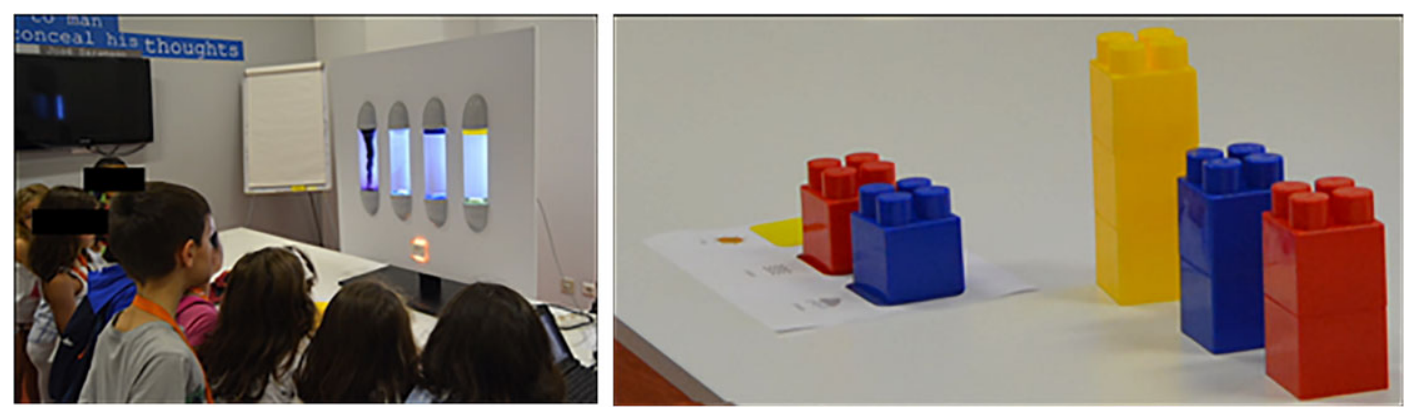

Figure 3. Left: Children observing the WISE's visualization; Right: Legos used in the interaction with children.

WISE information could be used in the day-to-day routines. The study ran under the supervision of two researchers: an interviewer and a note taker. Audio of the interviews was recorded.

\subsection{Participants and procedures EV2}

The second evaluation included a formal evaluation of children's knowledge concerning energy sources through a sixquestion questionnaire conducted after the interaction with the WISE installation. Five questions tested the children's knowledge about the local electricity production, the impact of different sources of energy on the environment and how the weather conditions impacted the electricity produced locally. One question probed the children about how enjoyable was the interaction with WISE.

The questions were constructed taking into account the limitation that small children might have when answering questionnaires (Read and Fine, 2005). For that matter, all questions had graphical and textual representations for the answers, and a psychologist member of the research team read the questions out loud and monitored the children along the way. The questions were explained to the group as a whole, however, each child responded individually, if needed there was a member of the research team present which could individually clarify any question. Table 1 lists the five questions presented in the questionnaire. Throughout the rest of this document, these questions will be referred as Q1, Q2, Q3, Q4 and Q5, respectively. The questionnaire was previously validated with a different group of four children with ages between 8 and 13 years old.

Two multiple choice questions (Q1 and Q2) asked children to select the types of energy produced locally and the types of electricity generation techniques which used renewable energy. Q3 inquired children about which production techniques are good or bad for the planet. Q4 presented children with three possible weather conditions (windy, sunny and rainy). Children were then asked to connect representations of the weather to pictures of four electricity generation techniques
Table 1. Five questions used in the evaluation with children.

\begin{tabular}{l} 
QD Question \\
Q1 \\
Select which energy sources you think are available in the \\
Island \\
Q2 Select which energy sources you think are renewable \\
Q3 Which energy sources are good and which are bad for the \\
Earth \\
Q4 Which energy sources are available when there is rain/sun/ \\
wind...? \\
Q5 Select which energy sources are available during the day \\
\hline
\end{tabular}

used in the Island. Children could assign one or more generation techniques to each weather condition based on how much that technique influenced the overall local load. Q5 presented children with a timeline of a day with different weather conditions. Children were asked to connect three points (morning, mid-day and night) to one or more of four electricity production techniques (Fig. 4).

About 56 children visited our research institute facilities during which visit they could try different demos, games and see exhibitions of research work, as well as interacting with the WISE installation. The evaluation of WISE installation was performed during such visit. To facilitate interaction, the 56 children (average 8.4 years old, 25 males) were organized into six groups based on their age:

- Group A: 7 kids between 10 and 12 years old

- Group B: 7 kids between 10 and 12 years old

- Group C: 11 kids between 8 and 9 years old

- Group D: 11 kids between 8 and 9 years old

- Group E: 11 kids between 6 and 7 years old

- Group F: 9 kids between 6 and 7 years old

The interaction with WISE was evaluated using a Wizard of $\mathrm{Oz}$ approach (Dow and MacIntyre, 2007). Each group had approximately 10 to $12 \mathrm{~min}$ with the installation followed by $10 \mathrm{~min}$ to fill in a questionnaire. Both the interaction and questionnaire sessions were video recorded. Figure 3 (left) shows the setup of the experiment. WISE was placed on a table and surrounded by participants, the Lego $^{\mathrm{TM}}$ blocks 


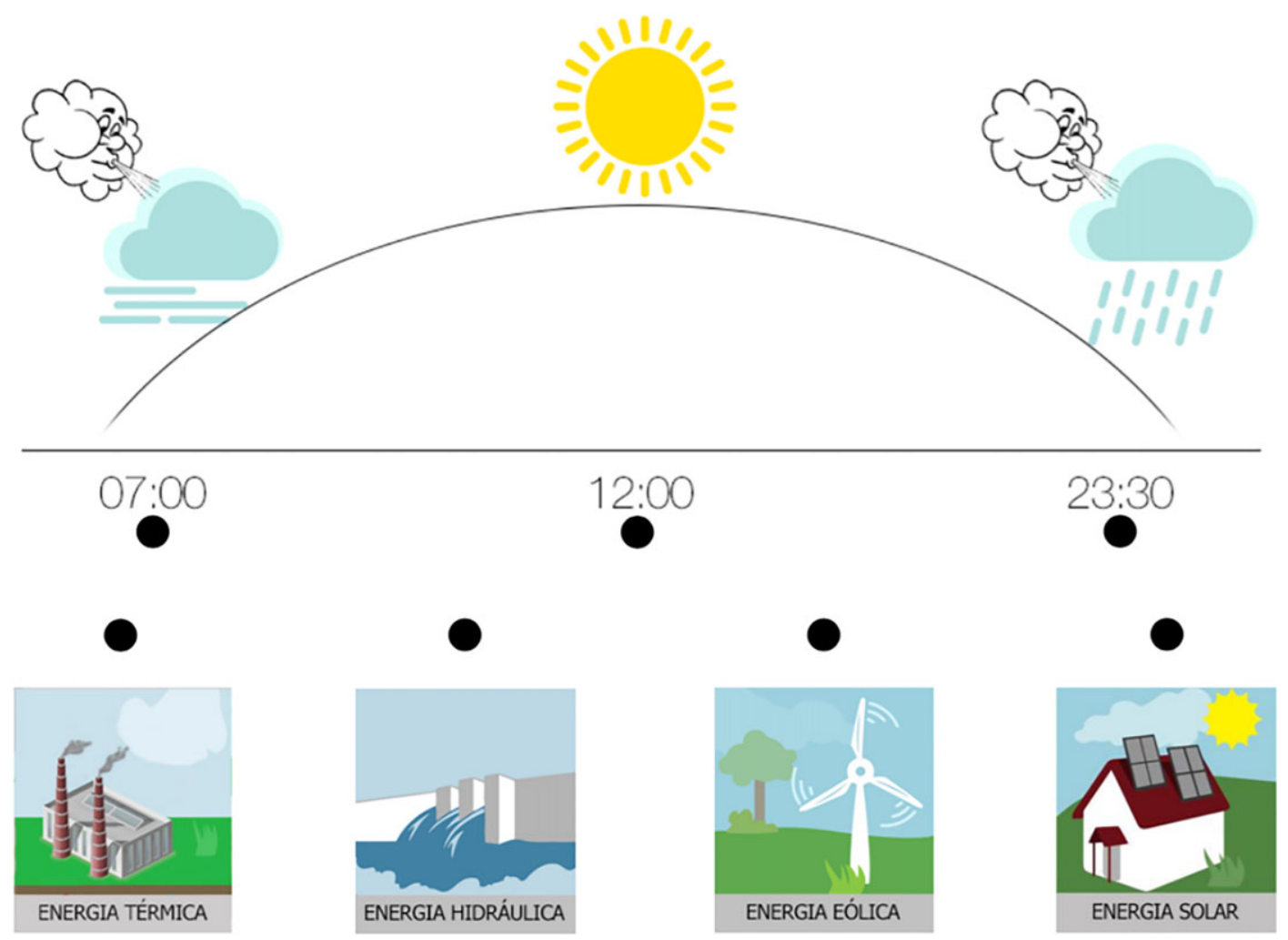

Figure 4. Fifth question of the children's evaluation of WISE. The black dots represent the locations children used to connect the images.

although not visible in Fig. 3 (left) were placed directly in front of the WISE panel.

\section{EVALUATION RESULTS}

In this section, results are summarized for both evaluations, organized in themes extracted from the qualitative assessment of both studies. More specifically, for EV2 qualitative data included behavioral data analysis such as verbal expressions, interaction patterns, behavior cues and gestures towards the installation, but also between the participants. This data was summarized through thematic analysis of observational notes drawn from videos taken during the installation and questionnaire session for EV2 (Braun and Clarke, 2006). Whenever relevant, quantitative results were added to this summary. In addition, whenever relevant, quotes were added to the results, namely, EV1 participants are referred as P1 to P10. For EV2, since the evaluation was done in groups, it was not possible to isolate individual reactions, as a result, participants quotes' in EV2 are not identified.

\subsection{EV1}

Concerning EV1, participants focused mostly on the information value provided by the installation. Overall, participants perceived it to be extremely useful and easy to interpret. Nevertheless, participants revealed misconceptions about energy sources, which became cleared out with the interaction. Below we explain further each of these elements.

\subsubsection{Usefulness of WISE}

In general adults' reactions to the system were pragmatic as they tried to gauge the value of a system such as WISE could bring to their own energy consumption monitoring strategy. All of them agreed on the usefulness of WISE. Seven participants commented how WISE information helps understanding one's impact on the environment. This observation was more evident when participants discussed the power socket, six participants affirmed that if they had to choose one functionality from WISE they would choose the colored power socket, due to its location at the point-of-interaction, where the consumption activity is happening. Participants also referred that the power socket provided enough information to make a decision (based on the color feedback), it was easy to understand and immediate, attracted attention and summarized the disaggregated information from the tubes in real-time.

Regarding the information displayed it was observed that participants had, in general, a wrong preconceived perception of the quotas of each energy used in the island, this way the interaction with WISE clarified individuals' understandings. Eight out of 10 participants expected solar power production 
to be higher. P10 commented how the island has a lot of solar panels and how on average it is a sunny place:

'I thought solar was higher since there is no need to direct sunlight to produce photo-voltaic energy'

Also, P3 commented regarding the average weather conditions in the island

'I though the sun [solar energy] would be stronger [higher quota] ...the island has a lot of solar panels... and a lot of sun (...)'.

Six participants expected wind power to be higher, again comments based on visual awareness as they relate the production directly to the high number of wind turbines existent in the island, as P6 commented:

'We could have also a bit more wind [higher quota for wind power] since we have so many [wind turbines] is could be put to better use.'

Overall, for some sources such as hydroelectric, participants commented on how very little they know about it, and how it is uncommon to see a hydroelectric station. As one participant (P3) referred, hydroelectric power might not be related to the 'now', but heavily influenced by the past weather conditions such as, if has been raining for the past days:

'I was not expecting the water energy... I did not even know we took advantage of enough hydroelectricity to feed into the grid... I thought it was insignificant'.

In conclusion, during the interview 5/10 participants expected a much higher production of renewable energy. Like P6 commented

'The others (renewable vortexes) are so low they should be used more often... the fact we do not use more natural resources... I was expecting more'

\subsubsection{Presentation of the production information}

Participants found the presentation of the data through the WISE interface itself attractive, easier to understand and provided a good notion of what is going on as it was fast to consult and intuitive. Six of the ten participants liked the colors representing each energy source, the movement of the vortex and how water closely relates to energy and the 'evil' color of the fossil energy source vortex. Three participants described WISE as more visible and 'realistic' commenting on the movement and water vortex appearing like energy or something powered by energy, Like P7 from EV1 mentioned.

'Numbers are not as memorable... if you asked me the numbers of my last electricity bill I cannot remember... I would more likely remember this'

However, six participants mentioned how it was less precise than graphs and numbers. Participants also suggested different additions to the installation that would help them get more information: a projection of resources to be used to produce energy; a version of WISE to be used on a mobile platform it was also referred that the same information could be displayed through bars of graphs to be easier to consult, as P2 suggested:

'It would be interesting, if we could have this in the smart-phone like a widget with the production of each one of the different energies, maybe using symbols or a bar chart to present the relationships between the quotas'

Participants also quickly associated the kinetic properties of the presentation with the information being shown, as participant P3 explained:

'...I like the colors [of the vortices], I like the fact that it is a whirlwind because it has to do with energy something always in movement, being produced (...) people need to know that it is energy and that energy manifests itself in many ways...it is something in movement or puts in movement.'

This property also helped to exacerbate the electricity production information as P7 commented, 'that looks worrying' commented when she realized that there was approximately $85 \%$ of the thermal (pollutant) energy being produced.

\subsubsection{Predicting Watt-I-See Adoption}

Results from the 20 questions survey allow us to foresee how WISE (or a similar system) could be used in individual's routines. Participants from EV1 displayed high interest on the importance of key feature and functionalities of Watt-I-See such as the ability to display disaggregated energy production sources and quotas, suggesting a greener consumption and forecasting production feedback through an interface. When asked how Watt-I-See could be used within the household participants rated as somewhat important the shift in activities to coincide with the availability of renewable energy (average 3.9, std.d 1.10). The forecast of the energy production was rated high regarding the importance of the feature in general as well as the importance of such information in consumers' daily routines (average 4.9, std.d 0.32 and average 4.2 std.d 0.79).

When asked about how the learned information would impact their day-to-day routines, at first most participants mentioned that they already do what they can to save energy, but knowing the source of the electricity could make them feel better if the renewable penetration is significant at any given moment, P5: 'I can't change... I will keep doing what I have to... but know I'm more pleased to know we used a lot of wind and hydro energy...'. However, in the end of the interaction, when participants became more familiar with the system they referred they could manage some of their routines to accommodate more renewables as p10 points out 'maybe with a perfect understanding of the weather conditions we could change those devices that aren't used every day, not the PC or TV'.

Additionally, participants also hypothesized how WISE information could be used in their daily lives five participants 
commented on mobiles phones as an 'easy to reach interface' that is always present (or near) consumers. Similarly, participants also commented about how WISE's information could be incorporated into other devices, as P2 commented: 'it could be interesting, if it was possible on the phone a widget with the production levels of each energy, using symbols and bars to show the relation among them'. It seems that a simple relation between different energy sources and its quotas provide enough renewable information for the average consumer's curiosity. To this point, other participants (P6) also mentioned that information about the thermal energy would be enough information 'I would probably have only the first tube because it is possible to know what we are doing wrong...while the others are good I do not need to worry about reducing them'. Regarding the possible long-term impact of interacting with WISE, 9 of the 10 participants felt confident they could estimate the availability of renewable energy based on time of day, season and weather conditions after the study.

\subsection{EV2}

The evaluation with children enhanced the importance of tangible interaction, as it allowed for children to understand the energy sources concepts' but also, the influence of weather conditions in energy production. Their perceptions were shaped by the tangible interaction and consequently, their performance in the questionnaire

\subsubsection{Interaction with WISE}

In general, when children interacted with the installation, they had frequent verbalizations with the researcher exploring scenarios and commenting on what they observed in the installation: 'Let's have more of this...' or 'Can we have one of each?' or even 'If we remove it becomes smaller/bigger'. The selection of the Lego ${ }^{\mathrm{TM}}$ blocks to control the WISE display was made collaboratively through frequent verbal interactions between the children closer to the installation and the ones further away. Overall, each group tried around six to eight different combinations. Children took turns interacting with the blocks and selecting a combination.

\subsubsection{Understanding of the energy sources}

We also assessed how the interaction with WISE affected children's knowledge of the local electricity production process via a post-study questionnaire. Next, we present a summary of the questionnaire results. Before analysing the questions individually, the count of correct answers was normalized (since certain questions have higher correct answers than others). Table 2 summarizes the results of the questionnaire, and Tables 3 and 4 present detailed results for each question. Next, we discuss the answers for each question in detail. In Q1 the most selected sources of energy in the Island were solar energy (53 out of 56 answers) and wind energy (45 out of 56 answers). The most common misconception was the absence of fossil-fuel (35 out of 56) and biomass generation (31 out of 56 answers) from the set of available local sources of energy. In Q2 the two most frequent accurate answers were selecting solar as a renewable energy source followed by not selecting nuclear (47 and 46 out of 56 answers, respectively). The most common misconception in the question was not mentioning biomass and hydroelectric energy as renewable energy sources (27 and 35 out of 56 answers, respectively). As we summarize in Table 2 there was a maximum of seven correct and seven incorrect answers to Q3. Fossil-fuel and Nuclear energy sources were selected,

Table 2. Average results organized by question of the post-study questionnaire with children compared to the maximum amount of correct and incorrect answers possible for each question.

\begin{tabular}{lcccc} 
Question & Correct answers & Average correct & Incorrect answers & Average incorrect \\
Q1 & 5 & 3,03 & 7 & 2,41 \\
Q2 & 6 & 3,03 & 7 & 2,46 \\
Q3 & 7 & 6,08 & 7 & 0,89 \\
Q4 & 6 & 3,76 & 6 & 2,35 \\
Q5 & 8 & 3,8 & 7 & 3,55 \\
\hline
\end{tabular}

Table 3. Answers for the first three questions from EV2 evaluation. The value on each cell represents the total of answer for each question.

\begin{tabular}{llcrrrrrr} 
& & Thermal & Solar & Hydro & Wind & Waves & Nuclear & Biomass \\
Q1 & Selected as available in the Island & 21 & 53 & 26 & 45 & 18 & 9 & 25 \\
& Not selected as available in the Island & 35 & 3 & 30 & 11 & 38 & 47 & 31 \\
Q2 & Selected as renewable & 14 & 47 & 29 & 39 & 30 & 10 & 21 \\
& Selected as not renewable & 42 & 9 & 27 & 17 & 26 & 46 & 35 \\
Q3 & Good for the planet & 52 & 9 & 6 & 52 & 3 & 0 & 10 \\
& Bad for the planet & 3 & 45 & 47 & 2 & 52 & 53 & 44 \\
\hline
\end{tabular}


Table 4. Answers for the last two questions from EV2 evaluation. The value on each cell represents the total of answer for each question. Table 1 lists all the questions used in EV2.

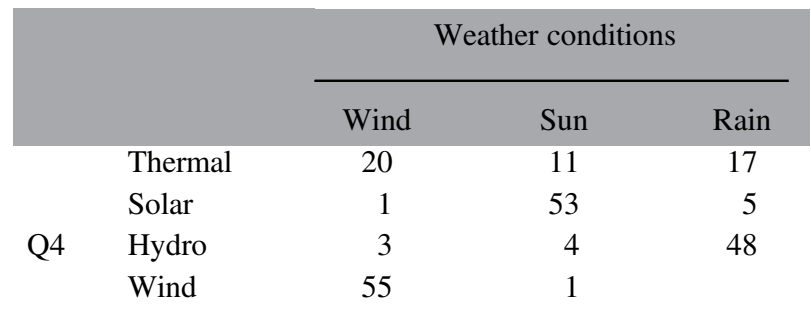

\begin{tabular}{rlrcc} 
& & \multicolumn{3}{c}{ Time of the day } \\
\cline { 3 - 5 } & & Morning & Mid-day & Evening \\
\multirow{2}{*}{ Q5 } & Thermal & 19 & 20 & 24 \\
& Solar & 9 & 7 & 42 \\
& Hydro & 44 & 5 & 12 \\
& Wind & 5 & 49 & 2 \\
\hline
\end{tabular}

52 out of 56, respectively, as bad for the planet. The most common misconception was selecting biomass and hydroelectric as bad for the environment ( 9 and 10 incorrect answers out of 56, respectively). Considering Q4 the most common misconception (71\% of the answers) was not to add the fossil-fuel energy option to all days since there is always a baseline (which sometimes is quite significant) of fossil-fuel energy produced locally. Finally, in Q5 (Fig. 4) 87\% of the participants identified mid-day as the best time of the day to produce photovoltaic energy. In fact, $70 \%$ of children could identify the morning as being the best time to produce hydroelectricity, mid-day to produce photovoltaic and night to produce hydro and wind electricity. The most common misconceptions in Q5 was not selecting wind energy available at night $(78 \%)$ and not adding fossil-fuel energy in the morning $(66 \%)$.

During the interaction with WISE, our observations of children verbalization's disclosed that they understood the energy sources as some children would reply to other children questions' or volunteer to explain that fossil-fuel was oil. One child even added: 'Oil is bad for the sea, the plants, and the environment'. Other children reacted to the combination a lot of rain and sun 'you have a rainbow'. The actual representation of energy also had an impact in children as they expressed amazement while observing the first demonstration 'This is cool!'. Children interrupted to ask questions and possible scenarios with 'how about this...?' or 'what happens if...?'.

\section{DISCUSSION}

In this section, we discuss the results from both evaluations. The discussion is organized around themes we found important throughout the study. Some themes combine results from both evaluations while others were unique for EV1 or for EV2.

\subsection{Usefulness of the production information in the daily lives}

When evaluating WISE with adults it was clear that participants started to think about how to include the provided information in their day-to-day routines, or how to change their routines to maximize the usage of one type of energy over the other. This way, one of the preferred aspects of WISE was feedback immediacy: The glass tubes and vortices provide just enough information to inform a decision. The feedback in the power socket is as close to the point-of-interaction as possible, however, at times, not the most visible due to the location of the power socket.

Adults also hypothesized scenarios of usage of a tool such as WISE, participants referred several constraints that could hinder shifting consumer behaviors according to the available electricity. Some daily or household activities cannot be shifted, like breakfast in the morning or dinner in the evening for example. However, some activities were mentioned as more flexible, such as washing machines and ironing (in line with Costanza et al. (2014) work). Yet, these activities are still affected by other factors such as weather conditions, for example using wind and sun to dry clothes naturally or using the washer and dryer machine. Withal, sporadic consumption routines were mentioned to be abler for shifting than daily routines. Extrinsic motivators such as monetary rewards from consumption reduction are not expressed through the WISE feedback. The study seemed to invoke more intrinsic motivators from the participants such as feeling worried or depressed when viewing the fossil vortex at its maximum scale.

This seems to suggest eco-feedforward incorporated into eco-feedback technologies enabled adult participants to better plan daily scenarios, and as a result, shift practices to use clean energies. However, it is important to mention that the results from WISE's interaction cannot be used to generalize how this information could be used in participants' daily routines. As it was mentioned in the introduction the novelty effect has been identified in eco-feedback systems (Nunes et al., 2011; Quintal et al., 2013b) and more recently also in eco-feedforward applications (Costanza et al., 2014). Researchers have approached this issue with automation, reducing the need for regular direct interaction with the system (Kobus et al., 2015). However, even autonomous systems need ways to display their internal state, and we believe our findings regarding the usefulness of the production information in the daily lives is a valuable contribution to such systems. Even considering a younger audience since our interaction and feedback strategies allowed children participants to extrapolate the environmental impact of energy sources being displayed after their time with WISE. 


\subsection{Energy visualization}

The visualization of the energy itself also had an impact on participants of both studies, we argue that the dramatic presentation of WISE not only fostered curiosity but also impacted the way participants viewed the information.

\subsubsection{EVI}

Regarding the adults, WISE was defined as a realistic representation of energy. Movement showed a significant effect on the engagement of participants. As a result, the simplistic representation of the energy production quotas thought the size of the colored vortex allowed for an attractive, understandable effect, that was suggested to be faster to consult and more intuitive. A stronger impact at times was noted. Participants felt somewhat worried when viewing the fossil vortex at its maximum scale how aggressive it looked. Others commented on the difference between fossils and renewable as depressing, something as needed to be dealt with.

\subsection{2. $\quad E V 2$}

Children saw the installation as something cool, they competed to see which one could interact with it. The ones that were not interacting with the installation were giving suggestions to their colleagues or commenting the results. In summary, our study showed that children enjoy tangible interaction when learning about complex concepts such as the correlation of naturally occurring phenomena and renewable energy. Results from our study suggest that WISE could work well as a complementary tool to science and energy education in schools. Not only to reinforce learned concepts (sun and wind as sources of renewable energy for example) but also to unveil the hidden or unclear ones (biomass and fossil-fuel energy baseline).

\subsection{Interacting with WISE}

\subsubsection{EV1}

Adults' interaction with WISE was also valuable, proven by the fact that most participants felt confident they could estimate the availability of renewable energy based on time of day, season and weather conditions after the study. We believe this confidence from consumers can be partially explained from the local setup, living in a close grid allows this type of extrapolation, which would not be possible in a continental location.

\subsection{2. $\quad$ EV2}

Considering the EV2, the experiment setup enabled up to nine different weather combinations, each group tried on averaged six different possibilities. This suggests the tangible interactive installation was highly successful in promoting exploration between naturally occurring phenomena and the availability of different forms of energy. This result is in line with the body of work which supports tangible interaction as a pedagogical tool (Antle et al., 2009; Bakker et al., 2011). We argue that these observations are justified by the tangibility and the familiarity of the raw resources used to produce renewable energy, and by the 'learning by doing' inherent quality of tangible interaction (Bakker et al., 2011; Zhang et al., 2010). Which in this case benefited the learning of variables that were more directly related to the tangible manipulation and detracted those who did not.

\subsection{Impact of production information}

Both adults (EV1) and children (EV2) were surprised when first exposed to the production information, as it defied preexistent notions of what kind of energy is available in the local network.

For less 'visible' renewable source productions such as hydroelectric consumers were mainly unaware of its impact on the grid. This observation is constant in both groups and we can argue that the visibility and everyday presence of the infrastructure used to produce solar (PV panels on the rooftops) and wind (windmill at the top of the mountains) electricity made participants overestimate its presence. On the other hand, hydroelectric stations are positioned in the base of the mountains away from residential areas, and this might be the cause for participants' misconceptions regarding hydroelectric energy. This argument is also valid to justify the overestimation of renewable energy in the island since the infrastructure used to produce thermal energy is located in an isolated place not accessible to 'normal' citizens.

Another misconception both in children and adults (EV1 and EV2) was an overestimation of the presence of renewable energy in the grid. Adults were expecting the natural resources were more used in the island. Children also overestimated the renewable quota by not mentioning the presence of a baseline of thermal (non-renewable) energy in the grid. This observation was clear from the answers of Q4 and Q5, which indicate that children do not understand that fossil-fuel is always used (at least locally) regardless of the penetration of renewable energy. More than two-thirds of respondents (67\%) failed to identify this phenomenon. This is, of course, related to the fact that fossilfuel is used to fill up the baseline of energy not met by renewable sources and not directly related to the naturally occurring phenomena manipulated by WISE. However, this is also how the electricity production process works and children (like probably many adults) totally miss this important consequence of energy production networks. This study has several limitations. The evaluation with children was conducted in groups, which might have carried a group effect reaction to the installation. Future studies would include conducting the study with children individually and assess for differences in responses. Although, when considering children studies, literature (Hanna et al., 2004) suggests children are more talkative and participate more in context groups. An additional limitation was associated with the fact that it would have been valuable to measure the effects 
before and after the interaction, in terms of knowledge acquired regarding energy sources. Future studies could adopt experiment design with random assignment and compare user's response before and after the intervention, or even use a control group to compare learning effects when using the installation versus a standard class where users could learn about energy availability and forecast. Future studies could as well, include a follow-up to assess children attitudes in the long term in what concerns their energy behaviors.

\section{CONCLUSIONS}

With WISE, we explored a possible future for eco-feedback devices through an installation that presented individuals with the origin of their electricity. WISE was developed as a probe that we used to evaluate how adults and children perceive electricity and the electric grid. Both groups found value in different parts of WISE, adults were more pragmatic and suggested ways of including production information into their routines. On the other hand, children were more curious on seeing the visualization in different stages and engaged in the visualization itself.

Another common observation was an overestimation of the renewable presence in the grid, children were completely unaware of the thermal energy baseline which is always present, while adults thought the natural resources in the island were used more. Another consistent observation was a general unfamiliarity of hydroelectricity in the island. We connected both observations with the physical presence of the grid infrastructure in everyone's lives, which might have caused both adults and children to overestimate its presence in the total quota.

WISE's also allowed participants to quickly understand the relationship between the weather and the available energy, and this study is another evidence that energy consumers grasp this concept as the way of connecting to the produced electricity. Withal our studies have shown collectively that, although both children and adults found interest in knowing more about the efforts to produce electricity, there are still several misconceptions regarding the grid operation. Our study exposed consumers to a disruptive scenario to which the electric grid is certainly approaching. Moreover, we decided to divide our study into two user studies in order to better cater for two very different target users, adults, who have power in the household, and children, since research has shown the importance of including children in energy savings techniques in the household (Barreto et al., 2014; Quintal et al., 2013a).

Regarding the interaction with WISE, we observed that the immediacy of the feedback in the power socket was one the favorite features of WISE, this observation supports literature findings which recommend that the feedback is most effective when presented close to the time of decisions and point-ofinteraction (Fischer, 2008). Individuals also referred that WISE kinetic representation of energy production helped deliver clear information about the electricity reaching one's home. This observation was true both for adults and children, consequently, we believe our approach of displaying the production in a metaphorical representation achieved its goal allowing us to study individuals' thoughts and reactions regarding the logic behind the electricity consumed in their daily lives. Overall the findings from these studies indicate promising directions for new forms of Eco Feed Forward systems.

\section{SUPPLEMENTARY MATERIAL}

Supplementary data are available at Interacting with Computers online.

\section{FUNDING}

This work was funded by the Fundação da Ciência e Tecnologia (FCT) grant number UID/EEA/50009/2013, and by the Agência Regional para o Desenvolvimento da Investigação Tecnologia e Inovação (ARDITI) grants number 002271/2014/131 and M14-20-09-5369-FSE-000001.

\section{REFERENCES}

Antle, A.N., Droumeva, M. and Ha, D. (2009). Hands on What?: Comparing Children's Mouse-based and Tangible-based Interaction. In Proc. 8th Int. Conf. Interaction Design and Children. ACM, pp. 80-88.

Bakker, S., van den Hoven, E. and Antle, A.N. (2011). MoSo Tangibles: Evaluating Embodied Learning. In Proc. 5th Int. Conf. Tangible, Embedded, and Embodied Interaction, TEI'11. ACM, New York, NY, USA, pp. 85-92. doi:10.1145/1935701.1935720.

Barreto, M.L., Szóstek, A., Karapanos, E., Nunes, N.J., Pereira, L. and Quintal, F. (2014) Understanding families' motivations for sustainable behaviors. Comput. Hum. Behav., 40, 6-15. doi:10. 1016/j.chb.2014.07.042.

Bourgeois, J., van der Linden, J., Kortuem, G., Price, B.A. and Rimmer, C. (2014). Conversations with My Washing Machine: An In-the-wild Study of Demand Shifting with Self-generated Energy. In Proc. 2014 ACM Int. Joint Conf. Pervasive and Ubiquitous Computing, UbiComp'14. ACM, New York, NY, USA, pp. 459-470. doi:10.1145/2632048.2632106.

Braun, V. and Clarke, V. (2006) Using thematic analysis in psychology. Qual. Res. Psychol., 3, 77-101. doi:10.1191/ 1478088706qp063oa.

Broms, L., Katzeff, C., Bång, M., Nyblom, Å.S.A., Hjelm, S.I. and Ehrnberger, K. (2010). Coffee Maker Patterns and the Design of Energy Feedback Artefacts. In Proc. 8th ACM Conf. Designing Interactive Systems, DIS'10. ACM, New York, NY, USA, pp. 93-102. doi:10.1145/1858171.1858191.

Chetty, M., Tran, D. and Grinter, R.E. (2008). Getting to Green: Understanding Resource Consumption in the Home. In Proc. 10th Int. Conf. Ubiquitous Computing, UbiComp'08. ACM, New York, NY, USA, pp. 242-251. doi:10.1145/1409635.1409668. 
Costanza, E., Fischer, J.E., Colley, J.A., Rodden, T., Ramchurn, S. D. and Jennings, N.R. (2014). Doing the Laundry with Agents: A Field Trial of a Future Smart Energy System in the Home. In Proc. SIGCHI Conf. Human Factors in Computing Systems, CHI'14. ACM, New York, NY, USA, pp. 813-822. doi:10.1145/ 2556288.2557167.

Dow, S. and MacIntyre, B. (2007). New Media Collaboration through Wizard-of $\mathrm{Oz}$ Simulations. In Computer-Human Interaction.

Ernevi, A., Jacobs, M., Mazé, R., Müller, C., Redström, J. and Worbin, L. (2005) The Energy Curtain: Energy Awareness. In Redström, J., Redström, M. and Mazé, R. (eds), IT+Textiles. (2010 reprint) Borås, Sweden: Centre for Textile Research (1st edn). pp. 91-96. IT Press/Edita, Helsinki.

European Commission. (2013). The EU climate and energy package.

Filonik, D., Medland, R., Foth, M. and Rittenbruch, M. (2013) A Customisable Dashboard Display for Environmental Performance Visualisations. In Berkovsky, S. and Freyne, J. (eds), Persuasive Technology, Lecture Notes in Computer Science. pp. 51-62. Springer Berlin Heidelberg.

Fischer, C. (2008) Feedback on household electricity consumption: a tool for saving energy? Energy Effic., 1, 79-104. doi:10.1007/ s12053-008-9009-7.

Fischer, J.E., et al. (2013). Recommending Energy Tariffs and Load Shifting Based on Smart Household Usage Profiling. Presented at the International Conference on Intelligent User Interfaces, pp. 383-394.

Froehlich, J., Findlater, L. and Landay, J. (2010). The Design of Eco-feedback Technology. In Proc. SIGCHI Conf. Human Factors in Computing Systems, CHI'10. ACM, New York, NY, USA, pp. 1999-2008. doi:10.1145/1753326.1753629.

Gustafsson, A. and Gyllenswärd, M. (2005). The power-aware cord: energy awareness through ambient information display. In CHI'05 Extended Abstracts on Human Factors in Computing Systems, CHI EA'05. ACM, New York, NY, USA, pp. 1423-1426. doi:10.1145/1056808.1056932.

Hanna, L., Neapolitan, D. and Risden, K. (2004). Evaluating Computer Game Concepts with Children. In Proc. 2004 Conf. Interaction Design and Children: Building a Community, IDC'04. ACM, New York, NY, USA, pp. 49-56. doi:10.1145/1017833. 1017840.

Heller, F. and Borchers, J. (2011). PowerSocket: Towards On-outlet Power Consumption Visualization. In CHI'11 Extended Abstracts on Human Factors in Computing Systems, CHI EA'11. ACM, New York, NY, USA, pp. 1981-1986. doi:10.1145/1979742. 1979901.

Heller, F., Tsoleridis, K. and Borchers, J. (2013). Counter Entropy: Visualizing Power Consumption in an Energy+ House. In CHI'13 Extended Abstracts on Human Factors in Computing Systems, CHI EA'13. ACM, New York, NY, USA, pp. 2363-2366. doi:10.1145/2468356.2468771.

Horn, M.S., Crouser, R.J. and Bers, M.U. (2012) Tangible interaction and learning: the case for a hybrid approach. Pers. Ubiquitous Comput., 16, 379-389.
International Energy Outlook 2016. (2016). US Energy Information Administration.

Ishii, H. and Ullmer, B. (1997). Tangible Bits: Towards Seamless Interfaces Between People, Bits and Atoms. In Proc. ACM SIGCHI Conf. Human Factors in Computing Systems, CHI'97. ACM, New York, NY, USA, pp. 234-241. doi:10.1145/258549. 258715.

Jönsson, L., Broms, L. and Katzeff, C. (2010). Watt-Lite: Energy Statistics Made Tangible. In Proc. 8th ACM Conf. Designing Interactive Systems, DIS'10. ACM, New York, NY, USA, pp. 240-243. doi:10.1145/1858171.1858214.

Kjeldskov, J., Skov, M.B., Paay, J., Lund, D., Madsen, T. and Nielsen, M., (2015). Eco-Forecasting for Domestic Electricity Use. In Proc. 33rd Ann. ACM Conf. Human Factors in Computing Systems, CHI'15. ACM, New York, NY, USA, pp. 1985-1988. doi:10.1145/2702123.2702318.

Kobus, C.B.A., Klaassen, E.A.M., Mugge, R. and Schoormans, J.P. L. (2015) A real-life assessment on the effect of smart appliances for shifting households' electricity demand. Appl. Energy, 147, 335-343. doi:10.1016/j.apenergy.2015.01.073.

Nunes, N.J., Pereira, L., Quintal, F. and Berges, M. (2011). Deploying and Evaluating the Effectiveness of Energy Ecofeedback Through a Low-cost NILM Solution. In Proc. 6th Int. Conf. Persuasive Technology. pp. 2-5.

Peschiera, G., Taylor, J.E. and Siegel, J.A. (2010) Response-relapse patterns of building occupant electricity consumption following exposure to personal, contextualized and occupant peer network utilization data. Energy Build, 42, 1329-1336. doi:10.1016/ j.enbuild.2010.03.001.

Pierce, J. and Paulos, E. (2010). Materializing Energy. In Proc. 8th ACM Conf. Designing Interactive Systems, DIS'10. ACM, New York, NY, USA, pp. 113-122. doi:10.1145/1858171.1858193.

Pierce, J. and Paulos, E. (2012a). Beyond Energy Monitors: Interaction, Energy, and Emerging Energy Systems. In Proc. SIGCHI Conf. Human Factors in Computing Systems, CHI'12. ACM, New York, NY, USA, pp. 665-674. doi:10.1145/ 2207676.2207771

Pierce, J. and Paulos, E. (2012b). The Local Energy Indicator: Designing for Wind and Solar Energy Systems in the Home. In Proc. Designing Interactive Systems Conference, DIS'12. ACM, New York, NY, USA, pp. 631-634. doi:10.1145/2317956.2318050.

Pierce, J. and Paulos, E. (2013). Electric Materialities and Interactive Technology. In Proc. SIGCHI Conf. Human Factors in Computing Systems, CHI'13. ACM, New York, NY, USA, pp. 119-128. doi:10.1145/2470654.2470672.

Quintal, F., Barreto, M., Nunes, N., Nisi, V. and Pereira, L. (2013a) WattsBurning on My Mailbox: A Tangible Art Inspired Ecofeedback Visualization for Sharing Energy Consumption. In Kotzé, P., Marsden, G., Lindgaard, G., Wesson, J. and Winckler, M. (eds), Human-Computer Interaction-INTERACT 2013, Lecture Notes in Computer Science. pp. 133-140. Springer Berlin Heidelberg.

Quintal, F., Pereira, L., Nunes, N., Nisi, V. and Barreto, M. (2013b) WATTSBurning: Design and Evaluation of an Innovative 
Eco-Feedback System. In Kotzé, P., Marsden, G., Lindgaard, G., Wesson, J. and Winckler, M. (eds), Human-Computer InteractionINTERACT 2013, Lecture Notes in Computer Science. pp. 453-470. Springer Berlin Heidelberg.

Read, J. and Fine, K. (2005). Using survey methods for design and evaluation in child computer interaction. In Workshop on Child Computer Interaction: Methodological Research at Interact.

Reading the clouds. (2008). Nuage Vert.

Schrammel, J., Gerdenitsch, C., Weiss, A., Kluckner, P.M. and Tscheligi, M. (2011) FORE-Watch-The Clock That Tells You When to Use: Persuading Users to Align Their Energy Consumption with Green Power Availability. In Keyson, D.V., Maher, M.L., Streitz, N., Cheok, A., Augusto, J.C., Wichert, R., Englebienne, G., Aghajan, H. and Kröse, B.J.A. (eds), Ambient Intelligence, Lecture Notes in Computer Science. pp. 157-166. Springer Berlin Heidelberg.

Simm, W., Ferrario, M.A., Friday, A., Newman, P., Forshaw, S., Hazas, M. and Dix, A. (2015). Tiree Energy Pulse: Exploring
Renewable Energy Forecasts on the Edge of the Grid. In Proc. 33rd Ann. ACM Conf. Human Factors in Computing Systems, CHI'15. ACM, New York, NY, USA, pp. 1965-1974. doi:10. $1145 / 2702123.2702285$.

Woodruff, A., Hasbrouck, J. and Augustin, S. (2008). A Bright Green Perspective on Sustainable Choices. In Proc. SIGCHI Conf. Human Factors in Computing Systems, CHI'08. ACM, New York, NY, USA, pp. 313-322. doi:10.1145/1357054. 1357109 .

Yang, R., Newman, M.W. and Forlizzi, J. (2014). Making Sustainability Sustainable: Challenges in the Design of Ecointeraction Technologies. In Proc. SIGCHI Conf. Human Factors in Computing Systems, CHI'14. ACM, New York, NY, USA, pp. 823-832. doi:10.1145/2556288.2557380.

Zhang, Z., Shrubsole, P. and Janse, M. (2010). Learning Environmental Factors Through Playful Interaction. In Proc. 9th Int. Conf. Interaction Design and Children, IDC'10. ACM, New York, NY, USA, pp. 166-173. doi:10.1145/1810543.1810562. 